\title{
Second Order Sliding Mode Control Scheme for an Autonomous Underwater Vehicle with Dynamic Region Concept
}

\author{
Zool H. Ismail and Vina W. E. Putranti \\ Centre for Artificial Intelligence \& Robotics, Universiti Teknologi Malaysia, Jalan Sultan Yahya Petra, \\ 54100 Kuala Lumpur, Malaysia \\ Correspondence should be addressed to Zool H. Ismail; zool@utm.my
}

Received 17 April 2015; Revised 17 June 2015; Accepted 18 June 2015

Academic Editor: Yong Liu

Copyright (C) 2015 Z. H. Ismail and V. W. E. Putranti. This is an open access article distributed under the Creative Commons Attribution License, which permits unrestricted use, distribution, and reproduction in any medium, provided the original work is properly cited.

\begin{abstract}
The main goal in developing closed loop control system for an Autonomous Underwater Vehicle (AUV) is to make a robust vehicle from natural and exogenous perturbations such as wind, wave, and ocean currents. However a well-known robust control, for instance, Sliding Mode Controller (SMC), gives a chattering effect and it influences the stability of an AUV. Furthermore, some researchers combined other controls to get better result but it tends to present long computational time and causes large energy consumption. Thus, this paper proposed a Super Twisting Sliding Mode Controller (STSMC) with dynamic region concept for an AUV. STSMC or a second order SMC is adopted as a robust controller which is free from chattering effect. Meanwhile, the implementation of dynamic region is useful to reduce the energy usage. As a result, the proposed controller obtains global asymptotic stability which is validated by using Lyapunov-like function. Moreover, some simulations present the efficiency of proposed controller. In conclusion, STSMC with region based control is effective to be applied for the robust tracking of an AUV. It contributes to give a fast response when handling the perturbations, short computational time, and low energy demand.
\end{abstract}

\section{Introduction}

The development of control stability for an Autonomous Underwater Vehicle (AUV) has gained much attention from many researchers since several years ago [1]. This happened because of the fundamental role of an AUV for replacing human involved in dangerous underwater activities for instance in underwater rescues, military purposes, underwater pipe inspections, oil and gas explorations, and so forth [2].

The robust tracking of an AUV against some natural disturbances is the main problem in this field $[3,4]$. Whereas a precision of tracking desire trajectory is important to be obtained, so that dissipation of energy can be avoided [5]. To cope with this problem, a range number of robust control systems have been introduced. Each control had its advantages and disadvantages. For this reason, many researchers coupled two or three different controllers or unified them with another control technique to improve its performance.
One example of conventional robust control was Proportional-Integral-Derivative (PID) controller [6]. It was a simple control but had difficulties not only for setting an appropriate value of its gain but also for reaching the expected requirement. Then, researchers combined PID with several methods such as PID with Fuzzy Control (FC), PID with selftuning technique, and PID with Genetic Algorithm (GA) [79]. The combination of PID with several methods focused on how to determine the gain value automatically. The control combination showed some improvements although there was a drawback like needed long computational steps which affected low real time execution.

Then, researchers adapted Linear Parameter Verifying (LPV) control and $H_{\infty}$ robust technique as the other methods $[10,11]$. However, the result of LPV control depicted good achievement only at minimum of sampling time, while $H_{\infty}$ robust technique presented smooth performance even though there was misstracking in some places. Furthermore, 
Sliding Mode Controller (SMC) was used as an alternative robust control. This control was commonly applied for an AUV [12-14]. Designing the closed loop control law of SMC based on Lyapunov candidate was required to remove the rule of linearization equation. Nevertheless, the main disadvantage of using this control was chattering effect. This negative effect appeared during the reaching condition and tended to be sensitive to the inaccurate mathematical model. In addition, chattering effect not only influenced the stability of an AUV but also generated large energy consumption. For this reason, some researchers proposed a dynamic region concept $[15,16]$. This method successfully reduced the energy usage.

Meanwhile to reduce the chattering effect, SMC was developed with other controls for instance SMC with fuzzy control or SMC with Neural Network (NN) [17-19]. Fuzzy control and $\mathrm{NN}$ were applied to tune the gain and to remove the nonlinearity from error dynamics, respectively. As a result, SMC with fuzzy control could achieve good parameter conditions after arranging many rules, while SMC with NN could perform satisfying condition after proceeding a lot of leanings and adaptations. Thus, this method required long data processes and consequently more energy demand will be spent, so that researcher expanded the formulation of SMC into Second Order Sliding Mode Control (SOSMC) [20-22]. SOSMC was aimed at removing the chattering effect which was produced by the conventional SMC. It worked on the second order of system deviation.

In this paper, Super Twisting Sliding Mode Controller (STSMC) with dynamic region concept for a 6-Degreeof-Freedom (DOF) holonomic AUV is proposed. STSMC ensures the robustness of an AUV when handling natural perturbation while energy consumption issue is overcame using region based concept. The global asymptotic stability of proposed control is analyzed by Lyapunov-like function. The rest of this paper is organized as follows. Section 2 describes the kinematics and dynamic properties of 6-DOF underwater vehicle. Section 3 states the robust control with dynamic region concept. The stability analysis in terms of the Lyapunov technique is also given in this section. Simulation results are presented in Section 4. Finally, Section 5 contains concluding remarks.

\section{Kinematics and Dynamics of a 6-DOF AUV}

The new formulation of robust control can be designed by considering the modeling system of a 6-DOF underwater vehicle. It involves a study on kinematics and dynamics system. Kinematics model is concerned with the equilibrium of the body at both rest and moving with certain velocity, while dynamics model is concerned with acceleration of the body motion. The studies of these were mainly discussed in [23].

2.1. Kinematic Model. The kinematics model has a correlation between inertial frame and body-fixed velocity of vehicle. It can be described by using the Jacobian matrix $J\left(\eta_{2}\right)$ in the following form [23]:

$$
\left[\begin{array}{l}
\dot{\eta}_{1} \\
\dot{\eta}_{2}
\end{array}\right]=\left[\begin{array}{cc}
J_{1}\left(\eta_{2}\right) & 0_{3 \times 3} \\
0_{3 \times 3} & J_{2}\left(\eta_{2}\right)
\end{array}\right]\left[\begin{array}{l}
v_{1} \\
v_{2}
\end{array}\right] \Longleftrightarrow \dot{\eta}=J\left(\eta_{2}\right) v,
$$

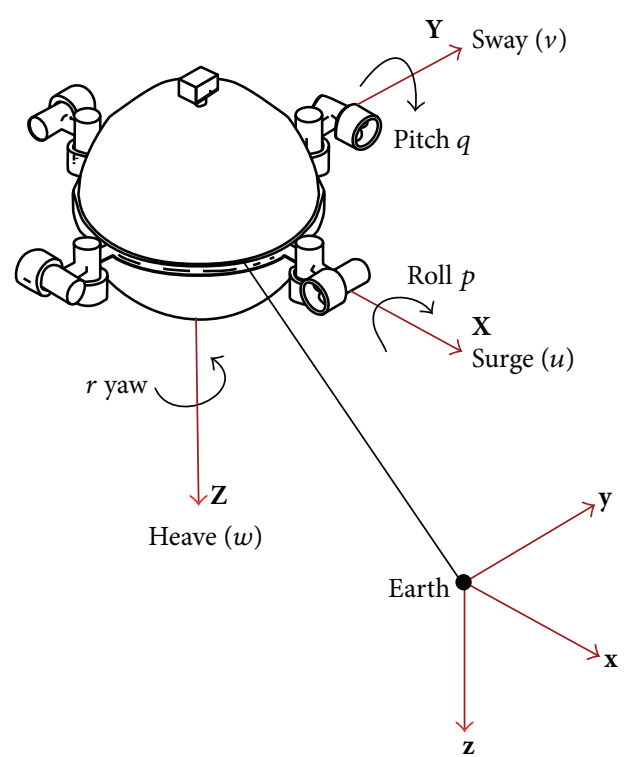

FIGURE 1: Body-fixed frame and earth fixed reference frame.

where $\eta_{1}=\left[\begin{array}{lll}x & y & z\end{array}\right]^{T} \in \mathbb{R}^{3}$ denotes the position while $\eta_{2}=$ $\left[\begin{array}{lll}\phi & \theta & \psi\end{array}\right]^{T} \in \mathbb{R}^{3}$ denotes the orientation of the vehicle which are expressed in the inertial-fixed frame. $J_{1}$ and $J_{2}$ are the transformation matrices described in Euler angles formation. Here, $v_{1}=\left[\begin{array}{lll}u & v & w\end{array}\right]^{T} \in \mathbb{R}^{3}$ and $v_{2}=\left[\begin{array}{ccc}p & q & r\end{array}\right]^{T} \in \mathbb{R}^{3}$ are the linear and angular velocity vectors, respectively, which are described in terms of the body-fixed frame relative to the earth's fixed frame. The illustration can be seen in Figure 1.

2.2. Dynamic Model. The dynamic equation of underwater vehicle can be expressed in closed form as

$$
M \dot{v}+C(v) v+D(v) v+g(\eta)=\tau .
$$

Equation (2) is changed with respect to the Jacobian matrix as the following equation [23]:

$$
M_{\eta}(\eta) \ddot{\eta}+C_{\eta}(v, \eta) \dot{\eta}+D_{\eta}(v, \eta) \dot{\eta}+g_{\eta}\left(\eta_{2}\right)=J^{-T} \tau,
$$

where $M_{\eta} \in \mathbb{R}^{6}$ is the inertia matrix including the added mass, $C_{\eta}(v, \eta) \in \mathbb{R}^{6}$ represents the matrix of the Coriolis and centripetal terms including the added mass, $D_{\eta}(v, \eta) \in \mathbb{R}^{6}$ denotes the hydrodynamic damping and lift force matrix, and $g_{\eta}\left(\eta_{2}\right) \in \mathbb{R}^{6}$ is vector of gravitational force and moment or the restoring force, while $\tau$ is the vector of generalized forces acting on the vehicle and can be written as the sum of estimated dynamics disturbances (control input).

There are numerous parameters which are required to be known. To reduce the complexity of the model, the dynamic equation in (3) preserves the following properties [23].

Property 1. The inertia matrix $M$ is symmetric and positive definite such that $M_{\eta}(\eta)=M_{\eta}(\eta)^{T}>0$.

Property 2. $C_{\eta}(v, \eta)$ is the skew-symmetric matrix such that $C_{\eta}(\nu, \eta)=-C_{\eta}^{T}(v, \eta)$. 
Property 3. The hydrodynamic damping matrix $D_{\eta}(v, \eta)$ is positive definite; that is, $D_{\eta}(v, \eta)=D_{\eta}{ }^{T}(v, \eta)>0$.

\section{Super Twisting Sliding Mode Control Scheme with Dynamic Region Concept}

The basic idea of super twisting sliding mode control with dynamic region concept is removing chattering effect, building a robust vehicle, and at the same time also saving the energy consumption. Before determining the proposed control, first it is necessary to construct a state space function of 6-DOF dynamic model:

$$
\begin{aligned}
x_{1} & =\eta \\
\dot{x}_{1} & =x_{2}, \\
x_{2} & =\dot{\eta}, \\
\dot{x}_{2} & =\ddot{\eta}=\left(J^{-T} \tau-\left(C_{\eta}(v, \eta) \dot{\eta}+D_{\eta}(v, \eta) \dot{\eta}+g_{\eta}\left(\eta_{2}\right)\right)\right) \\
& \cdot M_{\eta}^{-1}(\eta) .
\end{aligned}
$$

From (4), it can be reformed to be a matrix as

$$
\begin{aligned}
& \dot{x}=A x+B u, \\
& {\left[\begin{array}{c}
\dot{x}_{2} \\
\dot{x}_{1}
\end{array}\right]} \\
& =M_{\eta}^{-1}(\eta)\left[\begin{array}{cc}
-\left(C_{\eta}(v, \eta)+D_{\eta}(v, \eta)\right) & -g_{\eta} \\
1 & 0
\end{array}\right]\left[\begin{array}{l}
x_{2} \\
x_{1}
\end{array}\right] \\
& \quad+M_{\eta}^{-1}(\eta)\left[\begin{array}{l}
1 \\
0
\end{array}\right] \tau J^{-T} .
\end{aligned}
$$

Then, the proposed control is defined as

$$
\tau=\tau_{\text {st }}+\tau_{\text {eq }}
$$

where $\tau=$ force acting at the center mass of an AUV, $\tau_{\mathrm{st}}=$ super twisting control law, and $\tau_{\text {eq }}=$ equivalent control law.

From the above equation, final force consists of super twisting control $\left(\tau_{\text {st }}\right)$ which is added with equivalent control $\left(\tau_{\text {eq }}\right)$. After that, the equation $\tau_{\text {st }}$ is determined as the following step:

$$
\tau_{\mathrm{st}}=\tau_{1}+\tau_{2},
$$

where $\tau_{1}=$ discontinuous time derivative and $\tau_{2}=$ continuous function of sliding variable.

The value of derivative $\tau_{1}$ is

$$
\dot{\tau}_{1}=-W \operatorname{sgn}(s),
$$

where $s$ denotes a sliding vector and its value is proposed as

$$
s=\dot{\eta}-\dot{\eta}_{r}
$$

where $\dot{\eta}_{r}=$ virtual velocity (the value is formulated in the $\tau_{\text {eq }}$ process) and $W=$ control parameter.

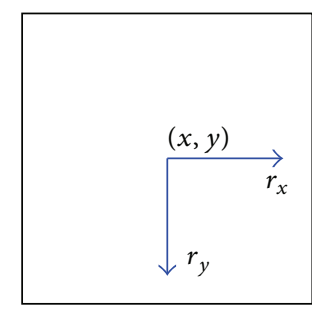

FIgURE 2: Rectangle desire region.

It is necessary to integrate (8) to get $\tau_{1}$. Then, formula of $\tau_{2}$ is determined as

$$
\tau_{2}=-k|s|^{0.5} \operatorname{sgn}(s),
$$

where $k$ is a constant value.

Remark 1. $\tau$ will converge to zero in finite time if $W$ and $k$ satisfy $W>\Phi / \Gamma_{M}$ and $k^{2} \geq 4 \Phi \Gamma_{M}(w+\Phi) / \Gamma_{m}^{2} \Gamma_{m}(W+\Phi)$, respectively. Here $\Gamma_{m}$ and $\Gamma_{M}$ are determined as $0 \leq \Gamma_{m} \leq$ $B(t, x) \leq \Gamma_{M}$.

Next is formulating the region boundary or $\tau_{\text {eq }}$. This concept begins by replacing the ordinary trajectory with a desire region. The step to determine the specific region can be seen as the following inequality function:

$$
f(\Delta \eta)=\left[f\left(\Delta \eta_{1}\right), f\left(\Delta \eta_{2}\right), \ldots, f\left(\Delta \eta_{N}\right)\right]^{T} \leq 0
$$

where $\Delta \eta=\left(\eta-\eta_{d}\right) \in \mathbb{R}^{6}, \eta_{d}=$ reference trajectory, and $N=$ total number of objective function.

For instance, the desire region is described as $2 \mathrm{D}$ with inequality function given in (12) and shown in Figure 2:

$$
\begin{aligned}
f\left(\Delta \eta_{1}\right)=\left(x-x_{d}\right)^{2}-r_{x}^{2} \leq & 0, \\
f\left(\Delta \eta_{2}\right)=\left(y-y_{d}\right)^{2}-r_{y}^{2} \leq & 0, \\
& \left(x-x_{d}\right)^{2}+\left(y-y_{d}\right)^{2} \leq r^{2},
\end{aligned}
$$

where $r$ is a regional bound which consists of $r_{x}$ and $r_{y}$.

After that, define the formula of potential energy for desire region. This step is useful as an energy consumption evaluation. The inequality function is described as

$$
\operatorname{EP}(\Delta \eta)= \begin{cases}0, & \text { if } f(\Delta \eta) \leq 0 \\ \frac{k_{\mathrm{ep}}}{2} f^{2}(\Delta \eta), & \text { if } f(\Delta \eta)>0\end{cases}
$$

Here, $k_{\text {ep }}$ denotes a positive constant. Note that when the AUV enters the bound or $f(\Delta \eta) \leq 0$, the gradient of $\operatorname{EP}(\Delta \eta)$ becomes smaller. 


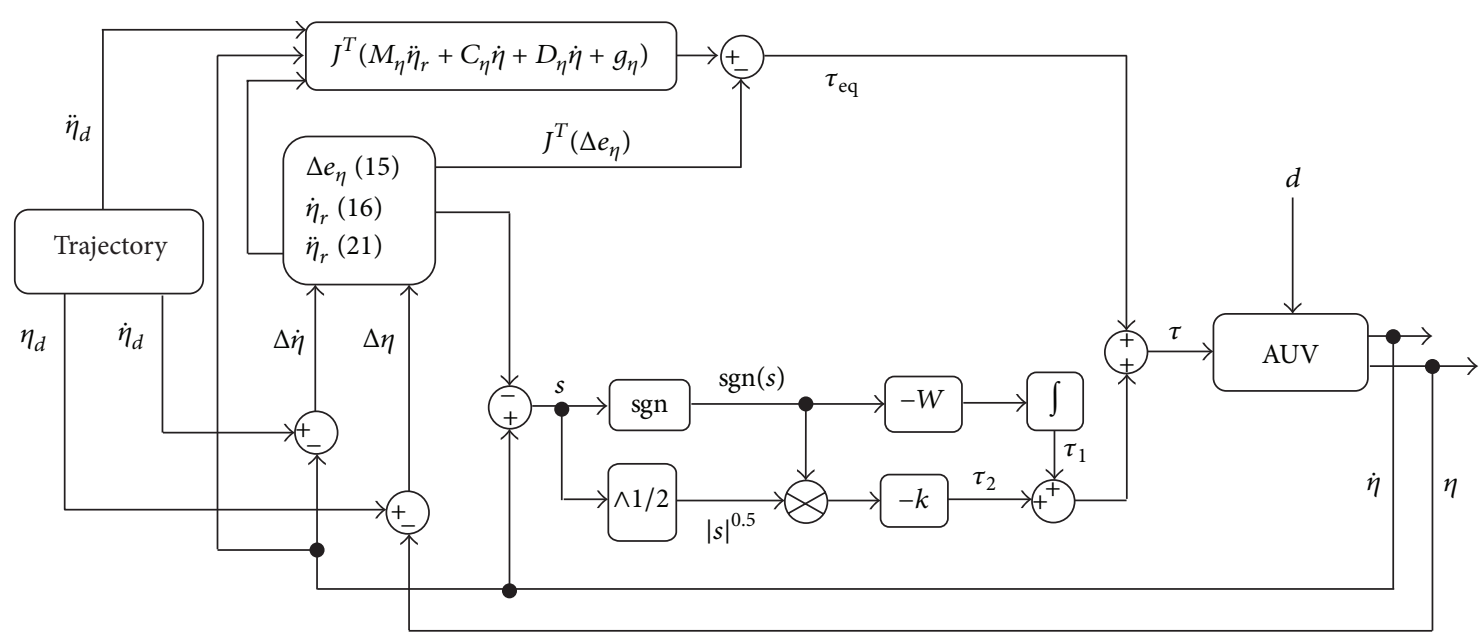

FIgURE 3: Proposed control scheme with dynamic region concept.

Then, the region error can be evaluated by partial differentiating (13) yielding

$$
\begin{aligned}
& \left(\frac{\partial \operatorname{EP}(\Delta \eta)}{\partial \Delta \eta}\right)^{T} \\
& = \begin{cases}0, & \text { if } f(\Delta \eta) \leq 0 \\
k_{\mathrm{ep}} f(\Delta \eta)\left(\frac{\partial f(\Delta \eta)}{\partial \Delta \eta}\right)^{T}, & \text { if } f(\Delta \eta)>0 .\end{cases}
\end{aligned}
$$

Therefore,

$$
\Delta e_{\eta}=k_{\mathrm{ep}} \max (0, f(\Delta \eta))\left(\frac{\partial f(\Delta \eta)}{\partial \Delta \eta}\right)^{T},
$$

where $\Delta e_{\eta}$ denotes region error.

Remark 2. $e_{\eta}$ triggers the AUV toward the desire region. Once the AUV is inside the region, the gradient of potential energy $\operatorname{EP}(\Delta \eta)$ becomes zero and at the same time $\Delta e_{\eta}$ reduces smoothly to zero.

Next step determines the value $\dot{\eta}_{r}$ based on the region error as

$$
\dot{\eta}_{r}=J^{-1}(\eta)\left(\dot{\eta}_{d}-\Delta \eta\right)-\alpha J^{-1}(\eta) \Delta e_{\eta} .
$$

Before formulating the final $\tau_{\text {eq }}$, it is needed to differentiate (9) and then multiply both sides with $M_{\eta}$ as in the following step:

$$
\begin{aligned}
\dot{s} & =\ddot{\eta}-\ddot{\eta}_{r}, \\
M_{\eta} \dot{s} & =M_{\eta} \ddot{\eta}-M_{\eta} \ddot{\eta}_{r} .
\end{aligned}
$$

From (3), the value of $M_{\eta} \ddot{\eta}$ is equal to $J^{-T} \tau-\left(C_{\eta} \dot{\eta}+D_{\eta} \dot{\eta}+g_{\eta}\right)$. Therefore, (18) changes to

$$
M_{\eta} \dot{s}=J^{-T} \tau-\left(M_{\eta} \ddot{\eta}_{r}+C_{\eta} \dot{\eta}+D_{\eta} \dot{\eta}+g_{\eta}\right) .
$$

Given $\dot{s}=0$, then the final equation of equivalent control is proposed as

$$
\tau_{\text {eq }}=J^{T}\left(M_{\eta} \ddot{\eta}_{r}+C_{\eta} \dot{\eta}+D_{\eta} \dot{\eta}+g_{\eta}\right)-J^{T} \Delta e_{\eta} .
$$

The value of $\ddot{\eta}_{r}$ is obtained by differentiating (16) as

$$
\begin{aligned}
\ddot{\eta}_{r}= & \dot{J}^{-1}(\eta)\left(\dot{\eta}_{d}-\Delta \eta\right)+J^{-1}(\eta)\left[\ddot{\eta}_{d}-\Delta \dot{\eta}\right] \\
& -\alpha \dot{J}^{-1}(\eta) \Delta e_{\eta}-\alpha J^{-1}\left(\Delta e_{\eta}+\Delta \dot{e}_{\eta}\right) .
\end{aligned}
$$

Remark 3. Equation of equivalent control $\left(\tau_{\mathrm{eq}}\right)$ can keep the state variables on the sliding surface by excluding the uncertainties in the dynamic equation.

The final proposed control equation (6) is transformed as

$$
\begin{aligned}
\tau= & \left(\int-W \operatorname{sgn}(s)\right)-k|s|^{0.5} \operatorname{sgn}(s) \\
& +J^{T}\left(M \ddot{\eta}_{r}+C \dot{\eta}+D \dot{\eta}+g\right)-J^{T} \Delta e_{\eta} .
\end{aligned}
$$

Theorem 4. The control law which is expressed in (22) with respect to the dynamic equation of an $A U V$ in (3) guarantees the global asymptotically stability of close-loop control system.

Proof. See Appendix.

All steps of super twisting sliding mode control with region boundaries are resumed in the general scheme as shown in Figure 3.

\section{Simulation Results}

The proposed control is applied on the ODIN vehicle (AUV) [24]. It is a 6-DOF holonomic AUV which has spherical shape. Further information can be seen in [24]. Here, an AUV is ordered to follow a straight red line trajectory as well as region trajectory. 


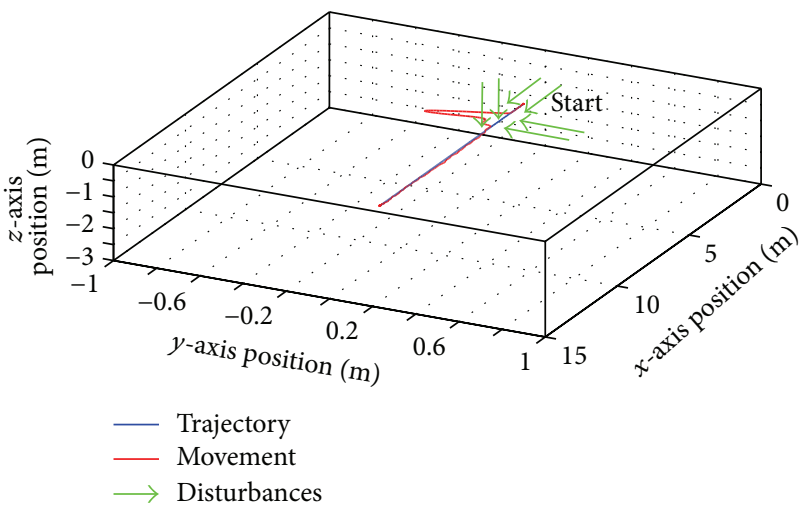

FIGURE 4: Tracking line of STSMC with line trajectory $(w=1, k=$ $3)$.

The region trajectory is defined as the following inequality:

$$
\begin{aligned}
f(\Delta \eta)= & \left(x-x_{d}\right)^{2}+\left(y-y_{d}\right)^{2}+\left(z-z_{d}\right)^{2} \\
& +\left(\phi-\phi_{d}\right)^{2}+\left(\theta-\theta_{d}\right)^{2}+\left(\psi-\psi_{d}\right)^{2} \leq r^{2}
\end{aligned}
$$

where $x, y$, and $z$ and $\phi, \theta$, and $\psi$ components represent the region and orientation specification, respectively, while $r \epsilon$ $\mathbb{R}^{6}$ is the error tolerance. Moreover, AUV starts from "start" sign.

In the middle of tracking activity, the AUV is suddenly attacked by some perturbations. The perturbations are given as velocity and their value is deterministic. Technical specifications of simulation are listed as follows:

$$
\begin{aligned}
& {\left[r_{x}, r_{y}, r_{z}\right] \in \mathbb{R}^{3}=0.1} \\
& {\left[r_{\phi}, r_{\theta}, r_{\psi},\right] \in \mathbb{R}^{3}=0.03} \\
& \text { Initial Point }=\left[\begin{array}{lll}
1.5 & 0 & -1.2
\end{array}\right]^{T} m \\
& \text { Initial Velocity }=\left[\begin{array}{lll}
1.5 & 0 & -1.2
\end{array}\right]^{T} \frac{m}{s} \\
& \text { Reaching Point }=\left[\begin{array}{lll}
10 & 0 & 0
\end{array}\right]^{T} m \\
& k_{\text {ep }}=10 \times 10^{-3} \\
& \alpha=10 \times 10^{-3} \\
& \text { Perturbations }=\left[\begin{array}{lll}
w x & w y & w z
\end{array}\right]^{T} \frac{m}{s} \\
& =\left[\begin{array}{lll}
0.08 & 0.08 & 0.08
\end{array}\right]^{T} \frac{m}{s},
\end{aligned}
$$

where $w x, w y$, and $w z$ are assumed as wind velocity, wave velocity, and ocean current velocity, respectively. Furthermore, the hitting period is about $10 \mathrm{~s}$ and exists in between $50 \mathrm{~s}$ and $60 \mathrm{~s}$ of time tracking.

First, proposed controller is employed under line trajectory. The results in Figures 4 and 5 showed that an AUV could track precisely on the line. However, its tracking performance

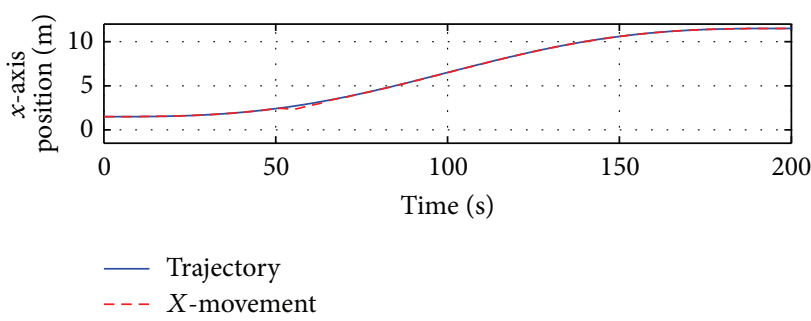

(a)

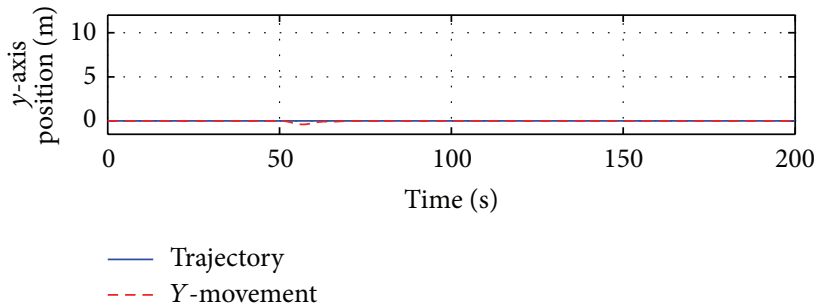

(b)

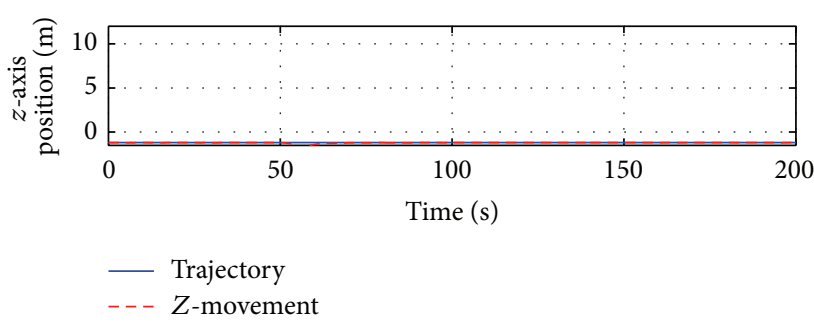

(c)

FIGURE 5: Linear position of STSMC with line trajectory.

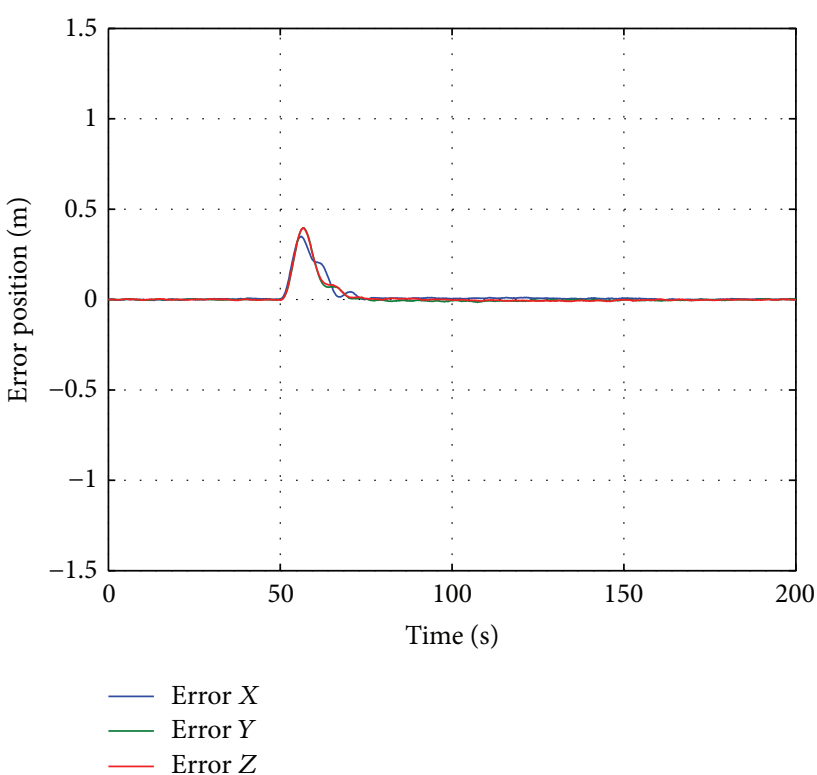

FIGURE 6: Error position of STSMC with line trajectory.

was disturbed after the presence of perturbation. Then, from Figure 6, it can be seen that an AUV needed 15 s to come back to desire trajectory. Meanwhile, the energy usage is presented in Figures 7 and 8. 


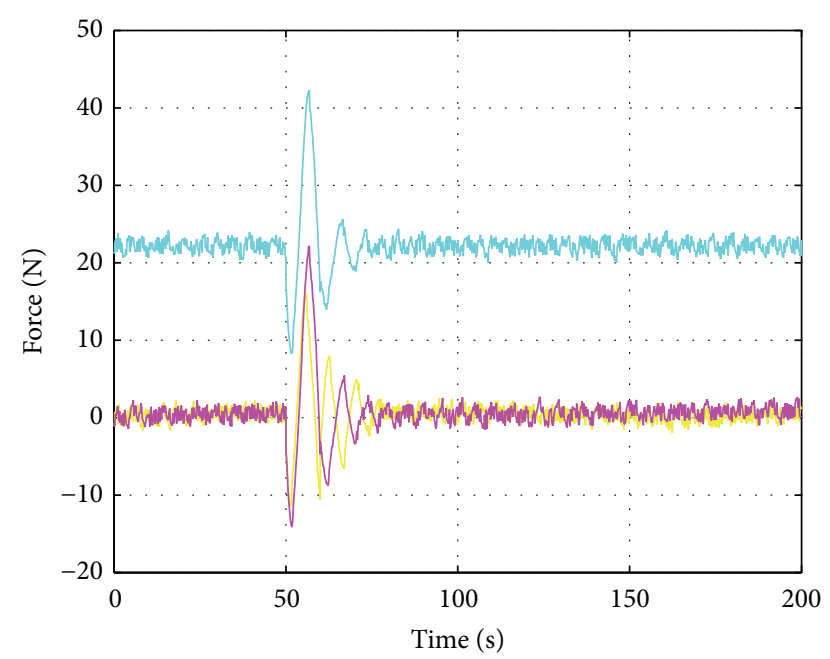

Force- $X$

- Force- $Y$

_ Force- $Z$

FIGURE 7: Forces of STSMC with line trajectory.

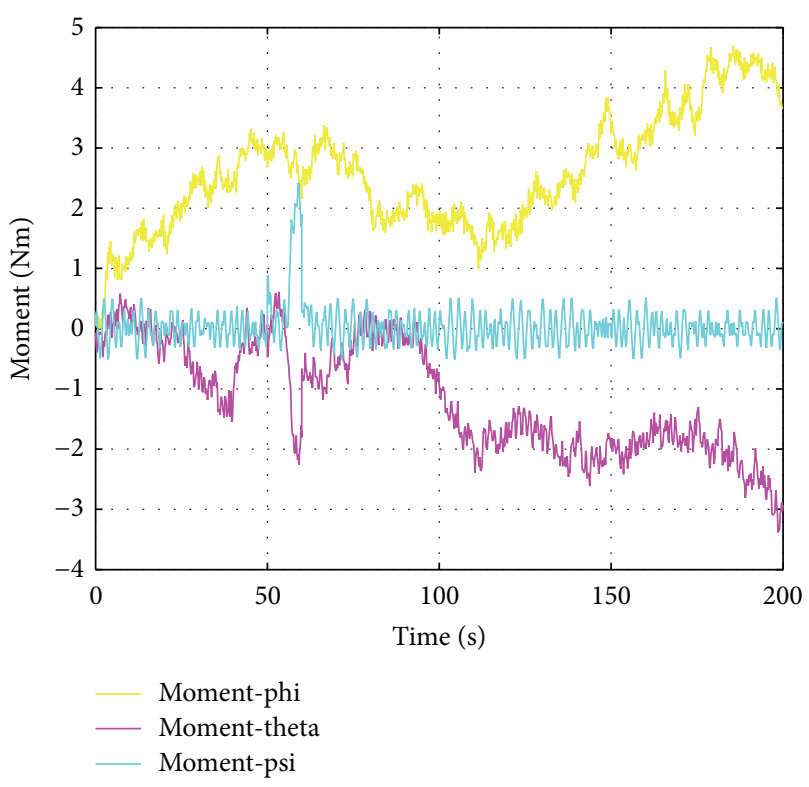

Figure 8: Moment of STSMC with line trajectory.

In the second case, line tracking trajectory is replaced by a region boundary. Its tracking performances are plotted in Figures 9 and 10. Although there were some disturbances, the AUV could keep on its position (inside the region). As a result, the amount of energy consumption is lower than in case 1 . Detailed information regarding error position and energy consumption can be seen in Figures 11-13.

To point out the differences with others, the proposed control is compared with adaptive control. An AUV with adaptive control requires tracking region trajectory. The existence of perturbations is the same as that in case 1 and case 2 . The results are performed in Figures 14-16. Without robust

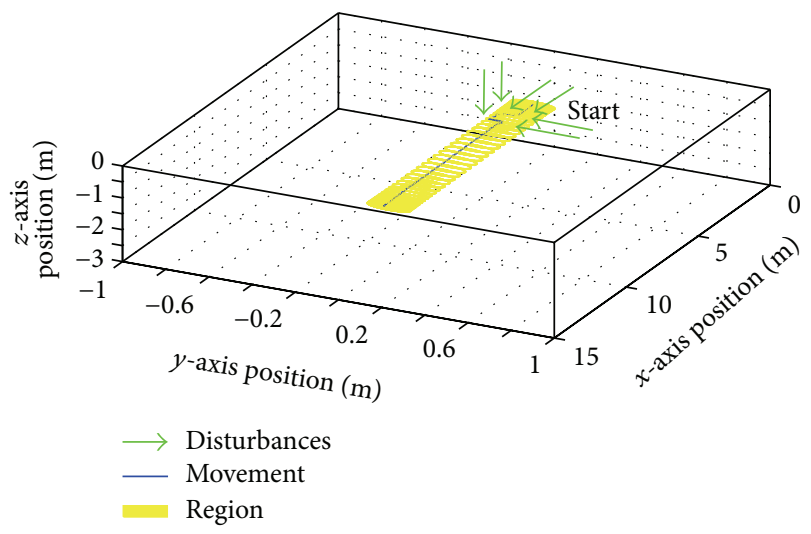

FIGURE 9: Tracking region of proposed control $(w=0.03, k=0.1)$.

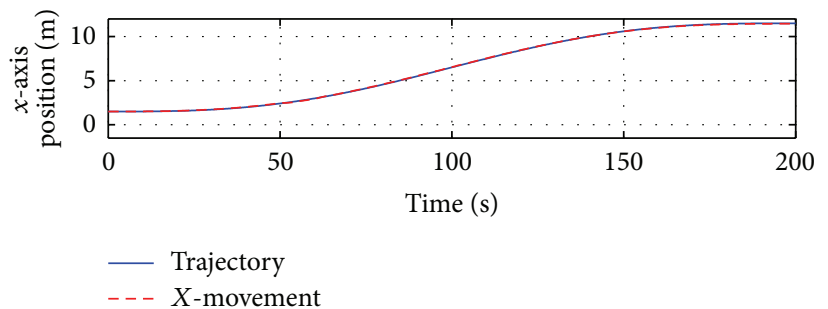

(a)

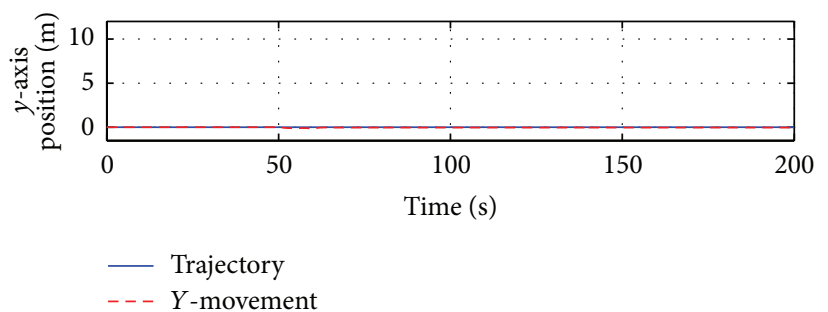

(b)

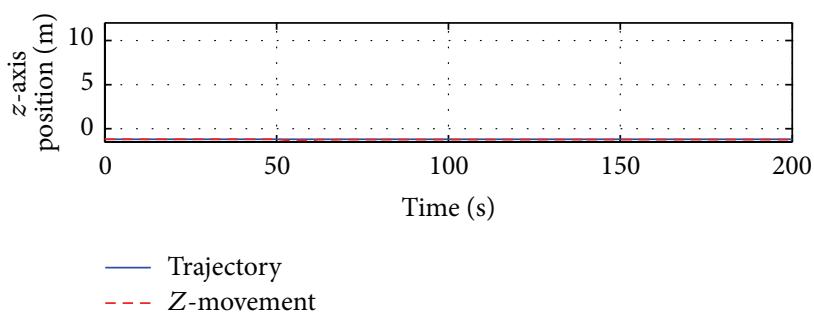

(c)

FIGURE 10: Linear position of proposed control with region trajectory.

control, an AUV moved out of the boundary and it also spent much energy to recover into desire condition. Graph of energy consumption is shown in Figures 17 and 18.

The total energy demand of each simulation is summarized in Table 1. Here, energy consumption is calculated from the forces and moment of each control. Total forces and moment are obtained by norm calculation for all the time. Less amount of energy consumption is produced under proposed controller. 


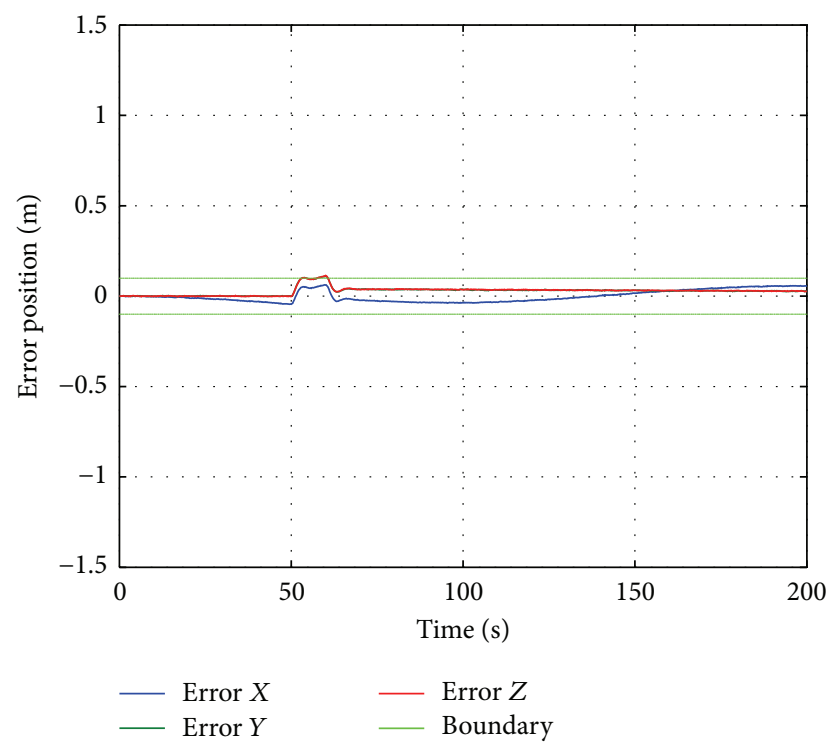

FIGURE 11: Error position of proposed control with region trajectory.

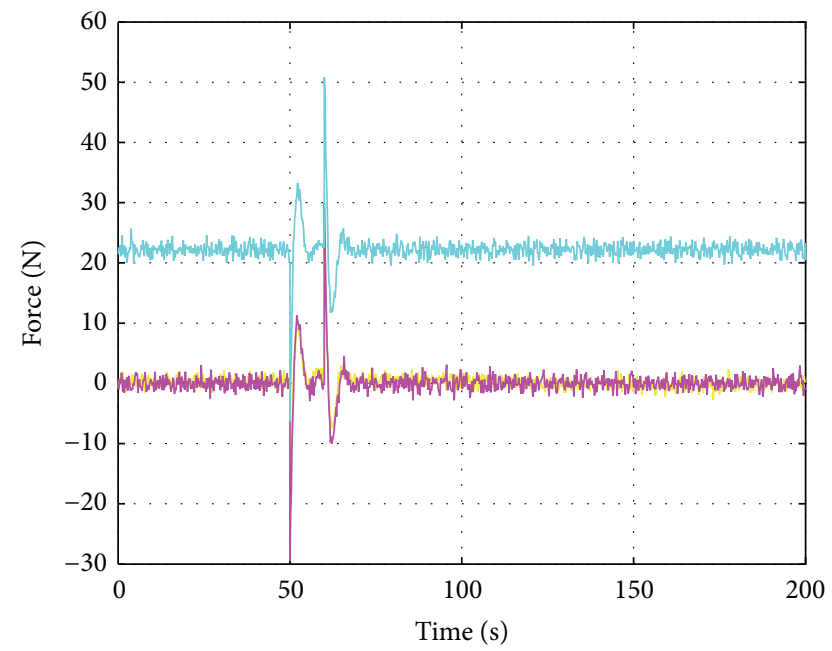

Force- $X$

- Force- $Y$

Force- $Z$

FIGURE 12: Forces of proposed control with region trajectory.

TABLE 1: Total energy consumption.

\begin{tabular}{lcc}
\hline Control & Force $(\mathrm{N})$ & Moment $(\mathrm{Nm})$ \\
\hline STSMC line trajectory & 711.77 & 96.23 \\
STSMC region trajectory & 709.30 & 7.47 \\
Adaptive region trajectory & 734.84 & 113.53 \\
\hline
\end{tabular}

Then, the propulsion of eight thrusters of ODIN can be described from total energy demand. The calculation is presented in the following formula:

$$
\tau=E F_{\text {th }} \text {, }
$$

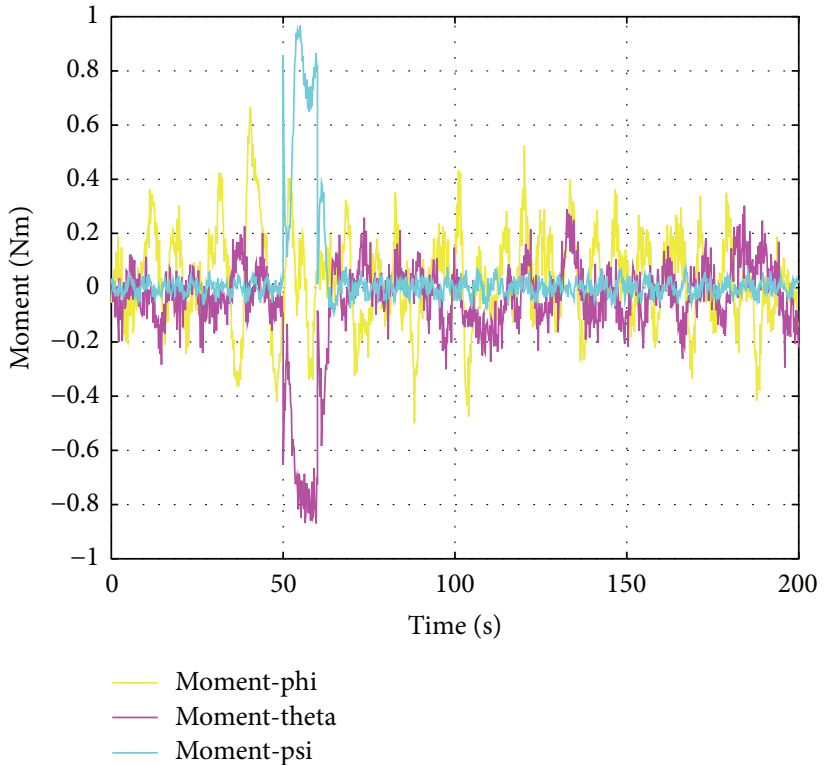

FIGURE 13: Moment of proposed control with region trajectory.

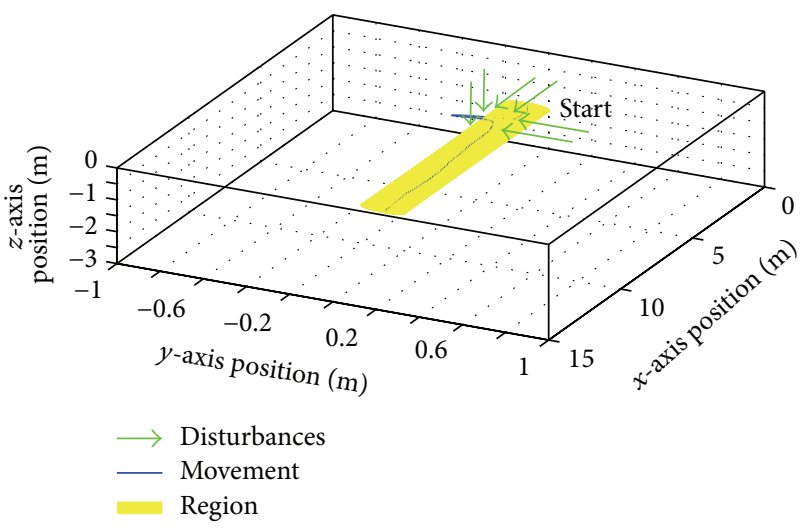

FIGURE 14: Tracking region of adaptive control.

where $\tau$ is given in (22) and $E$ denotes thrusters configuration matrix, while $F_{\text {th }}$ is vector of thrusters forces. To get thrusters propulsion $\left(F_{\text {th }}\right)$, it is necessary to inverse $E$ then multiplied by $\tau$. Here, the value of $E$ is equal to

$$
E=\left[\begin{array}{cccccccc}
c & -c & -c & c & 0 & 0 & 0 & 0 \\
c & c & -c & -c & 0 & 0 & 0 & 0 \\
0 & 0 & 0 & 0 & -1 & -1 & -1 & -1 \\
0 & 0 & 0 & 0 & R c & R c & -R c & -R c \\
0 & 0 & 0 & 0 & R c & -R c & -R c & R c \\
R_{z} & -R_{z} & R_{z} & -R_{z} & 0 & 0 & 0 & 0
\end{array}\right]
$$

where $c=\sin (1 / 4) \pi, R=0.381 \mathrm{~m}$, and $R_{z}=0.508 \mathrm{~m}$ representing the distance from center of vehicle to center of vertical thrusters and distance from center of vehicle to center of horizontal thrusters, respectively [25]. 


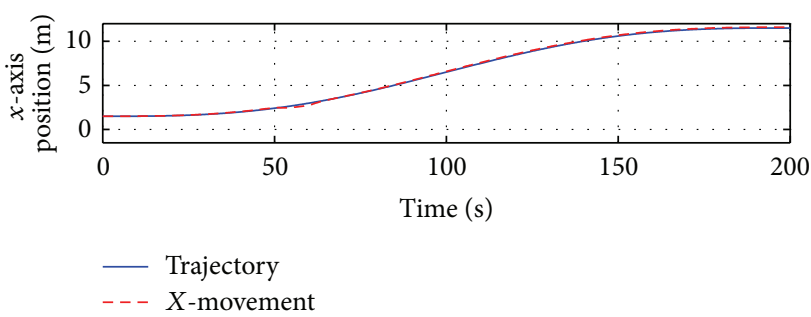

(a)

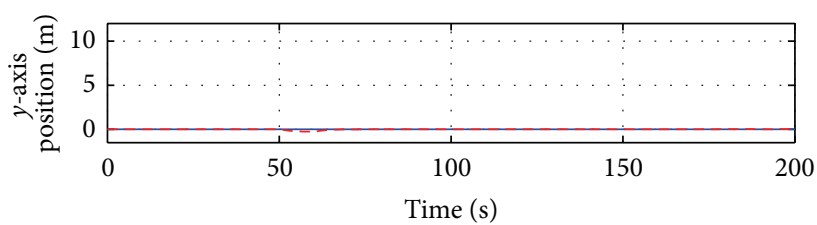

- Trajectory

- - - Y-movement

(b)

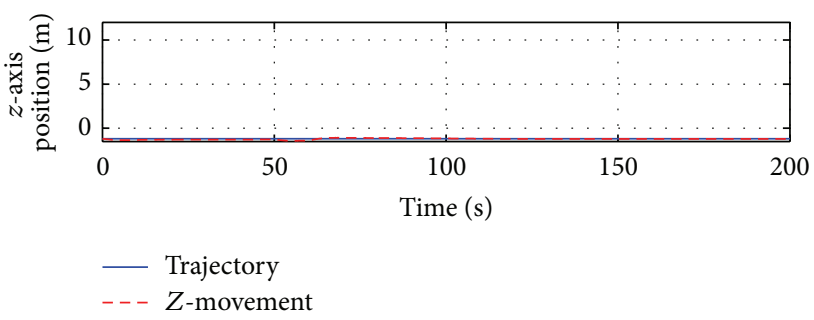

(c)

FIGURE 15: Linear position of adaptive control with region trajectory.

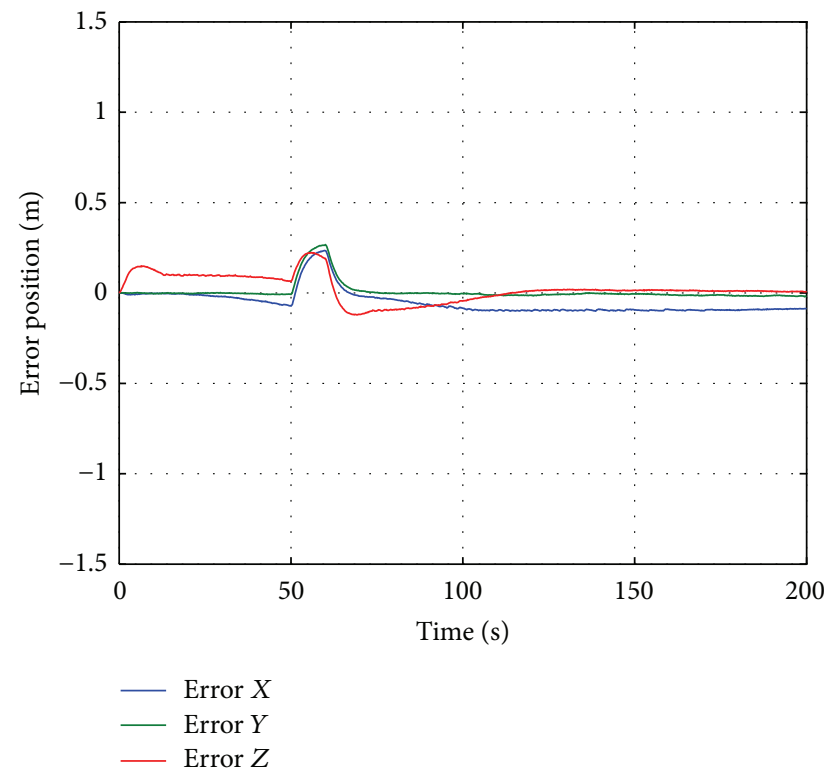

FIGURE 16: Error position of adaptive control with region boundaries.

Finally, the results of eight propulsions are performed in Figure 19 for propulsion in the case of STSMC with line trajectory and Figure 20 for propulsion in the case of STSMC with region trajectory, while Figure 21 is depicted for thruster's

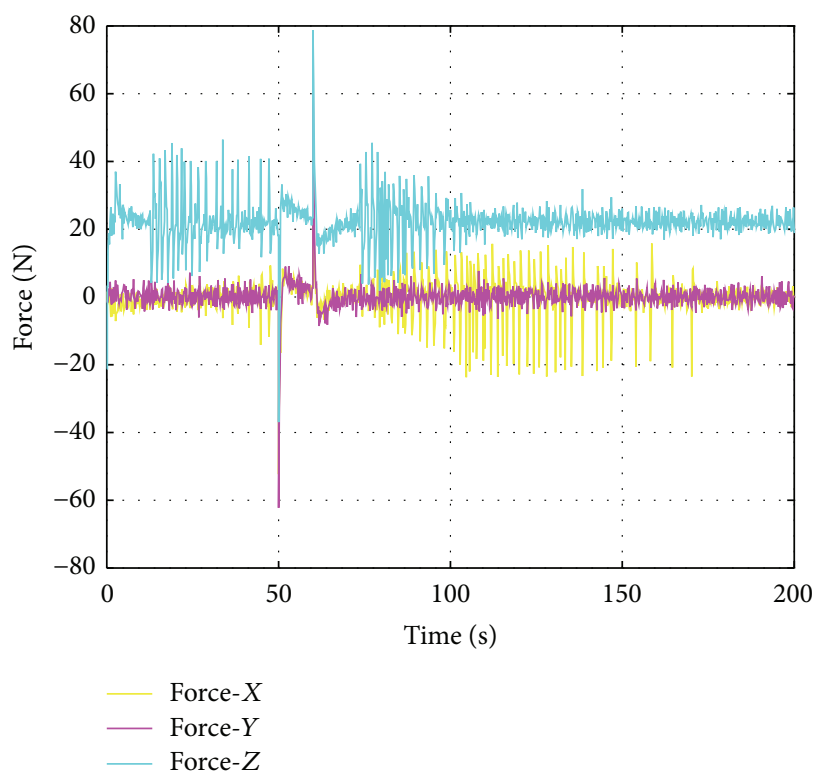

FIGURE 17: Forces of adaptive control with region boundaries.

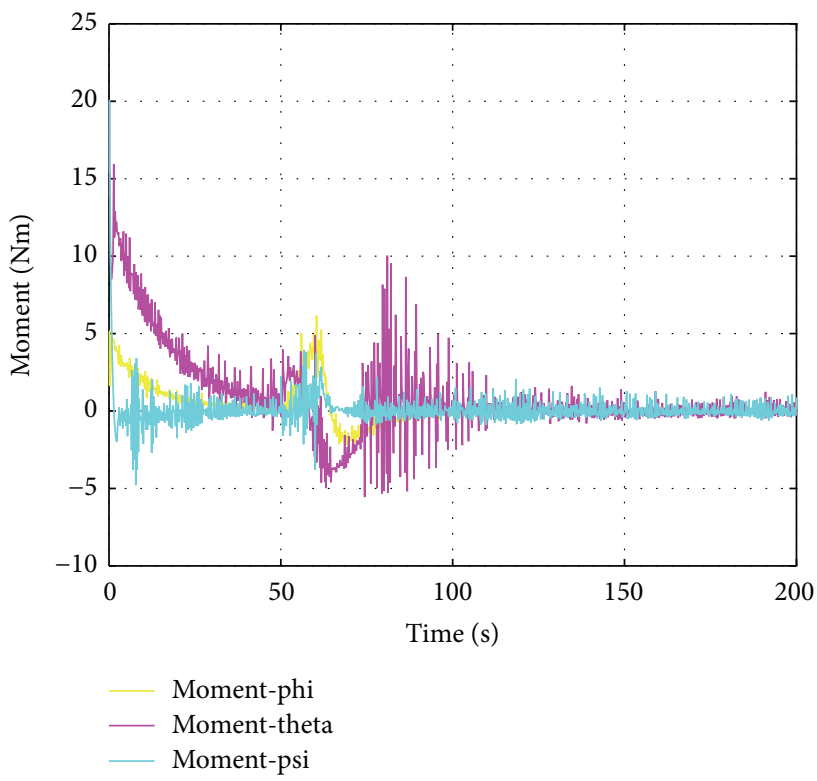

FIGURE 18: Moment of adaptive control with region boundaries.

propulsion in adaptive control with region trajectory. The figures indicate that more forces are spent by thrusters when some perturbations hit the AUV (started in 50 s). It means that, in those conditions, thrusters tried to keep the AUV's position from its desire trajectory. Compared to all methods, adaptive control required the most thruster forces followed by STSMC with line trajectory. Furthermore, the least thruster's propulsion is needed by proposed controller, STSMC with dynamic region (in Figure 20). Thrusters 2 and 4 generated around $0 \mathrm{~N}$ forces although the perturbations disturbed its movement. These results are not performed by another control. 

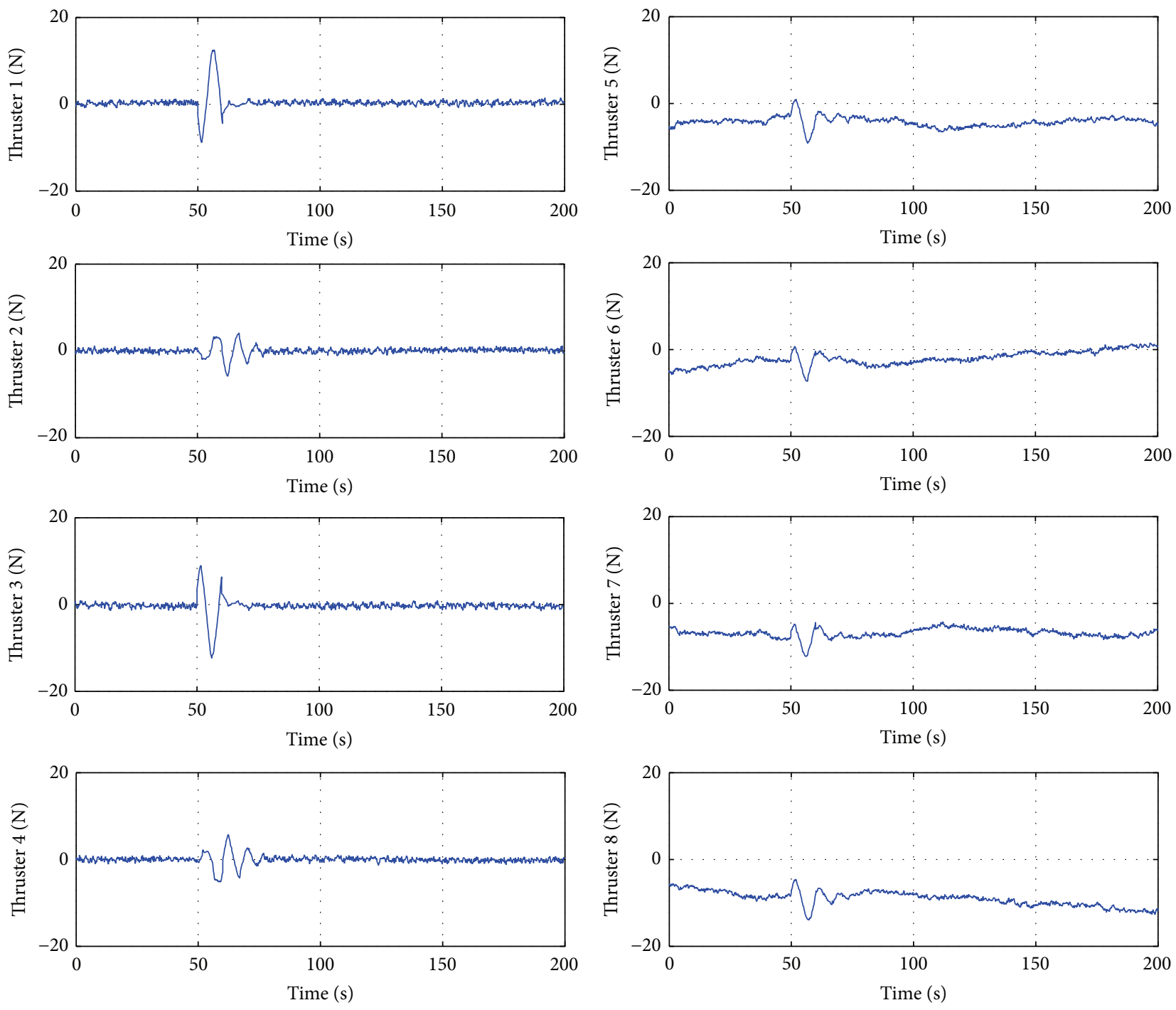

(a)

(b)

FIGURE 19: Thruster forces using STSMC with line trajectory. (a) Horizontal thrusters and (b) vertical thrusters.

\section{Conclusion}

A new robust control scheme based on second order Sliding Mode Controller with dynamic region is proposed in this paper. Super twisting is adapted as a second order sliding mode which is useful to make a robust AUV while region boundaries technique is applied as energy reduction. Some simulations were arranged to observe the effectiveness of proposed controller. In case 1, the AUV can track line trajectory under perturbations even though there are small errors positions. Then in case 2, the proposed control can maintain the AUV's position inside the region even if it is disturbed by the same amount of perturbations. Meanwhile, in the last case, the AUV moves out of the region and produces large error. From all results, it can be concluded that proposed control can save more energy consumption than others although the tracking movements are not precise in the middle of region. The amount of energy can be reduced as long as an AUV is inside the region.

\section{Appendix}

Define a positive definite function that satisfy as a Lyapunov candidate as below,

$$
\begin{aligned}
V= & \frac{1}{2} s^{T} M_{\eta} s \\
& +k_{\mathrm{ep}} \max (0, f(\Delta \eta))(\Delta \dot{\eta})^{T}\left(\frac{\partial f(\Delta \eta)}{\partial \Delta \eta}\right)^{T} \\
& +2 k|s|+\frac{1}{2} \tau_{1}{ }^{2}+\frac{1}{2}\left(W|s|^{1 / 2} \operatorname{sgn}(s)-\tau_{1}\right)^{2} .
\end{aligned}
$$



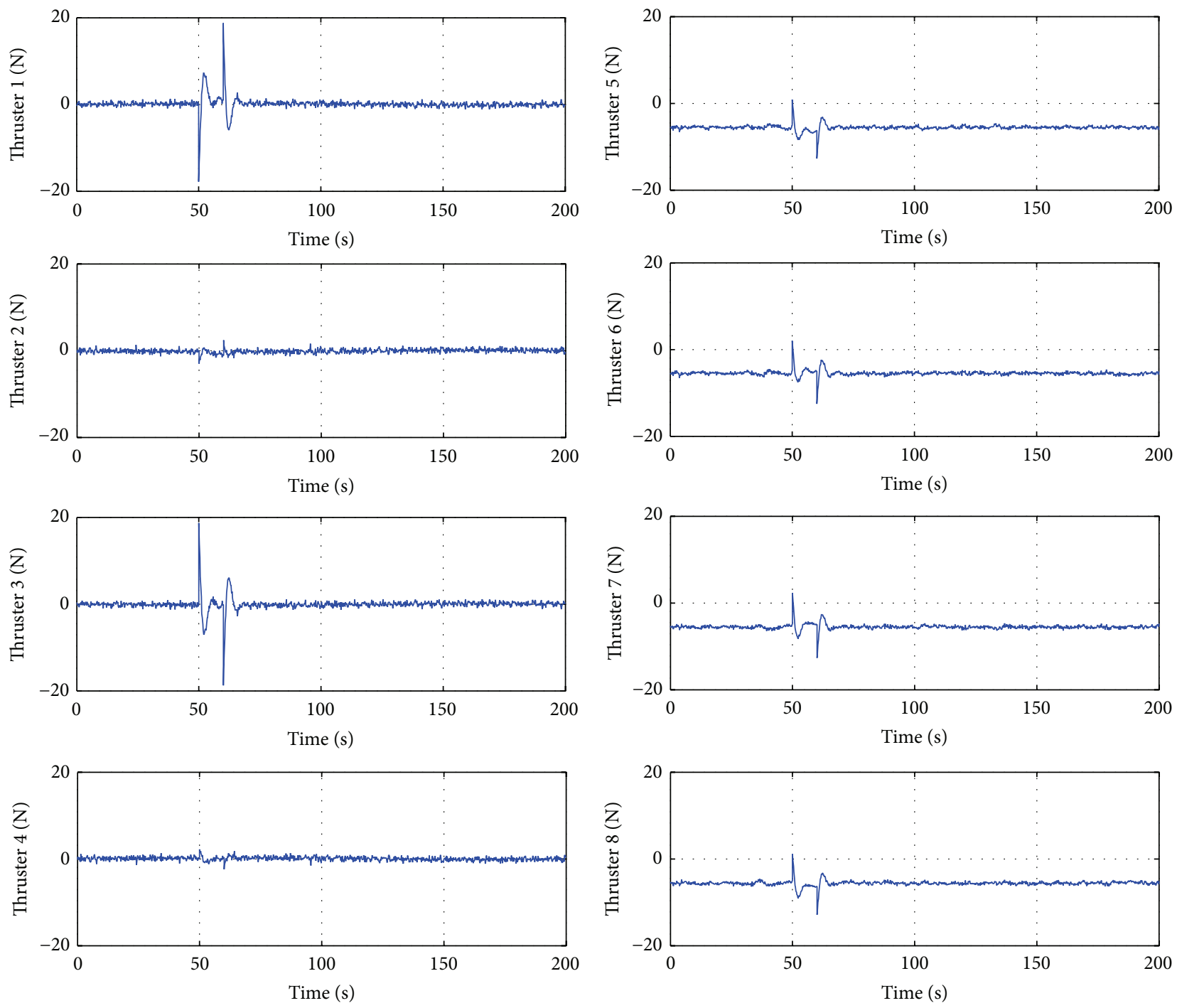

(a)

(b)

FIGURE 20: Thruster forces using STSMC with region trajectory. (a) Horizontal thrusters and (b) vertical thrusters.

Next, replace $2 k|s|+(1 / 2) \tau_{1}{ }^{2}+(1 / 2)\left(W|s|^{1 / 2} \operatorname{sgn}(s)-\tau_{1}\right)^{2}$ by $\zeta^{T} P \zeta$, where $\zeta^{T}=\left(|s|^{1 / 2} \operatorname{sgn}(s) \tau_{1}\right)^{T}$ and, $P=$ $(1 / 2)\left(\begin{array}{c}4 k+W^{2}-W \\ -W\end{array}-2\right)$.

Then, differentiating $V$ with respect to time yields

$$
\begin{aligned}
\dot{V}= & \frac{1}{2}\left(s^{T} \dot{M}_{\eta} s+\dot{s}^{T} M_{\eta} s+s^{T} M_{\eta} \dot{s}\right) \\
& +k_{\mathrm{ep}} \max (0, f(\Delta \eta))(\Delta \dot{\eta})^{T}\left(\frac{\partial f(\Delta \eta)}{\partial \Delta \eta}\right)^{T} \\
& -\frac{1}{\left|s^{1 / 2}\right|} \zeta^{T} Q \zeta+\frac{f(t, s)}{\left|s^{1 / 2}\right|} q_{1}{ }^{T} \zeta,
\end{aligned}
$$

where $Q=(W / 2)\left(\begin{array}{c}2 k+W^{2}-W \\ -W\end{array}\right)$ and $q_{1}{ }^{T}=\left(2 k+(1 / 2) W^{2}-\right.$ $(1 / 2) W)$.
Now apply $\dot{s}^{T} M_{\eta} s=s^{T} M_{\eta} \dot{s}$. Thus, $\dot{V}$ becomes

$$
\begin{aligned}
\dot{V}= & \frac{1}{2}\left(s^{T} \dot{M}_{\eta} s+2 s^{T} M_{\eta} \dot{s}\right) \\
& +k_{\mathrm{ep}} \max (0, f(\Delta \eta))(\Delta \dot{\eta})^{T}\left(\frac{\partial f(\Delta \eta)}{\partial \Delta \eta}\right)^{T} \\
& -\frac{1}{\left|s^{1 / 2}\right|} \zeta^{T} Q \zeta+\frac{f(t, s)}{\left|s^{1 / 2}\right|} q_{1}^{T} \zeta, \\
\dot{V}= & \frac{1}{2} s^{T}\left(\dot{M}_{\eta}-2 C_{\eta}\right) s+s^{T} M_{\eta} \dot{s} \\
& +k_{\mathrm{ep}} \max (0, f(\Delta \eta))(\Delta \dot{\eta})^{T}\left(\frac{\partial f(\Delta \eta)}{\partial \Delta \eta}\right)^{T} \\
& -\frac{1}{\left|s^{1 / 2}\right|} \zeta^{T} Q \zeta+\frac{f(t, s)}{\left|s^{1 / 2}\right|} q_{1}^{T} \zeta .
\end{aligned}
$$



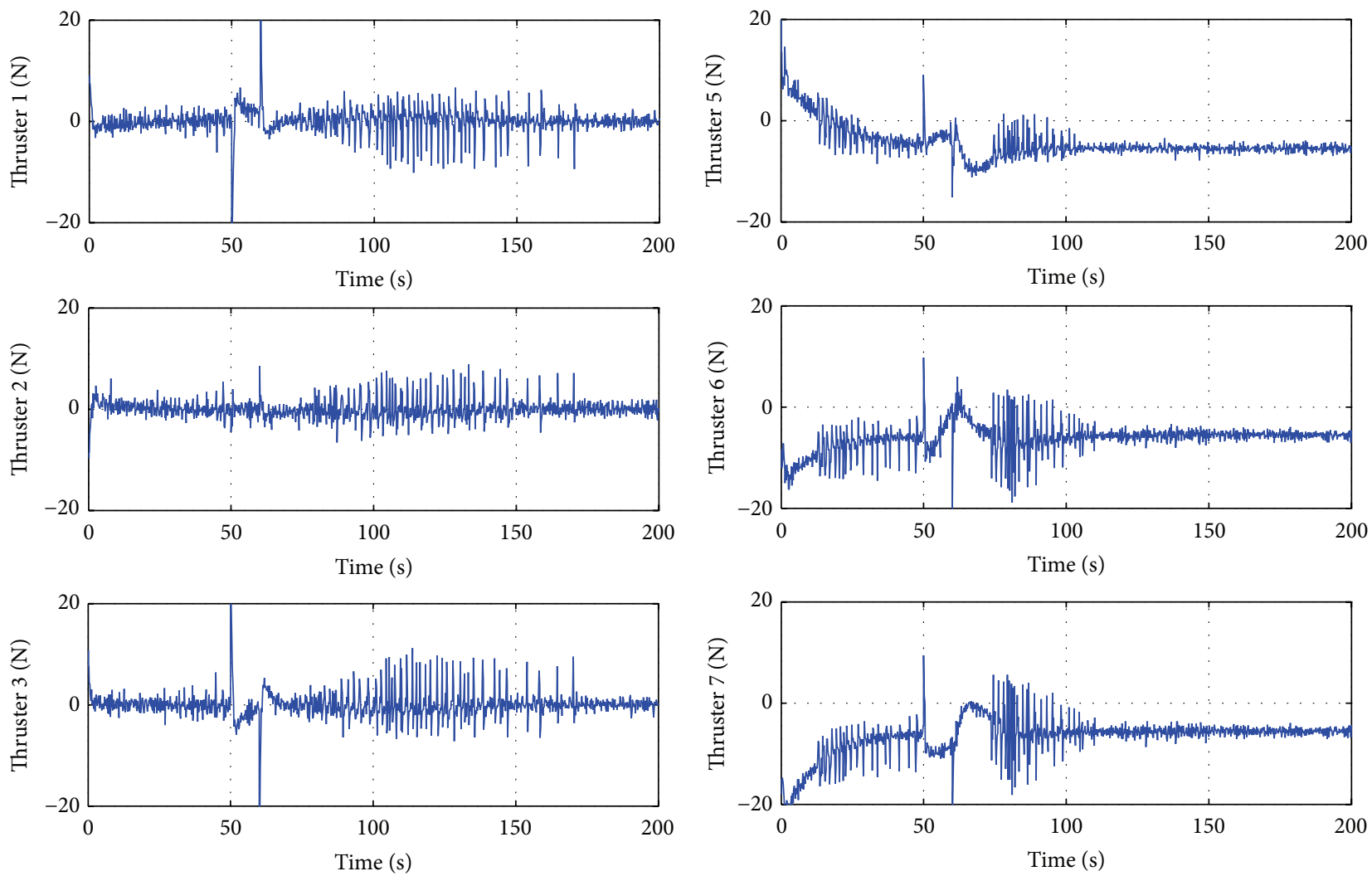

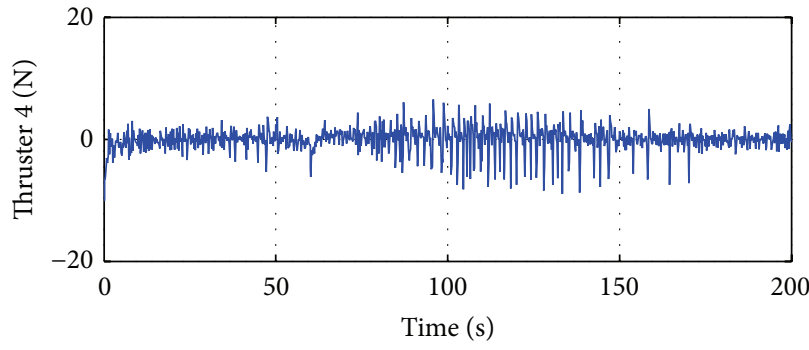

(a)

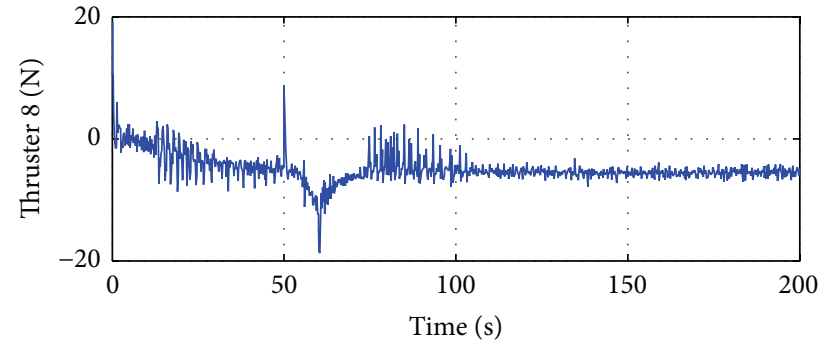

(b)

FIGURE 21: Thruster forces using adaptive control with region boundary. (a) Horizontal thrusters and (b) vertical thrusters.

Note that $s^{T}\left(\dot{M}_{\eta}-2 C_{\eta}\right) s$ is a skew symmetric matrix; therefore, its value is equal to zero (Property 2) [23]. Next, use the component $M_{\eta} \dot{s}=J^{-T} \tau-\left(M_{\eta} \ddot{\eta}_{r}+C_{\eta} \dot{\eta}+D_{\eta} \dot{\eta}+g_{\eta}\right)$ from (18), then combined with the perturbation which is given in [26] to get a globally bounded of (7):

$$
\begin{aligned}
\dot{V}= & s^{T}\left(J^{-T} \tau-\left(M_{\eta} \ddot{\eta}_{r}+C_{\eta} \dot{\eta}+D_{\eta} \dot{\eta}+g_{\eta}\right)\right) \\
& +k_{\mathrm{ep}} \max (0, f(\Delta \eta))(\Delta \dot{\eta})^{T}\left(\frac{\partial f(\Delta \eta)}{\partial \Delta \eta}\right)^{T} \\
& -\frac{W}{2\left|s^{1 / 2}\right|} \zeta^{T} Q \zeta,
\end{aligned}
$$

where $\delta=$ coefficient of perturbation and $Q=$ $\left(\begin{array}{cc}2 k+W^{2}-(4 k / W+W) \delta & -W+2 \delta \\ -W+2 \delta & 1\end{array}\right)$.
Hereafter, let $M_{\eta} \ddot{\eta}_{r}+C_{\eta} \dot{\eta}+D_{\eta} \dot{\eta}+g_{\eta}=F$. Now substitute $\tau_{\mathrm{eq}}=J^{T}(F)-J^{T} \Delta e_{\eta}$ from (19) to $\tau$ in the above equation:

$$
\begin{aligned}
\dot{V}= & s^{T}\left(J^{-T}\left(J^{T}(F)-J^{T} \Delta e_{\eta}\right)-F\right) \\
& +k_{\text {ep }} \max (0, f(\Delta \eta))(\Delta \dot{\eta})^{T}\left(\frac{\partial f(\Delta \eta)}{\partial \Delta \eta}\right)^{T} \\
& -\frac{W}{2\left|s^{1 / 2}\right|} \zeta^{T} Q \zeta
\end{aligned}
$$

or

$$
\begin{gathered}
\dot{V}=-s^{T}\left(\Delta e_{\eta}\right)+k_{\mathrm{ep}} \max (0, f(\Delta \eta))(\Delta \dot{\eta})^{T} \\
\cdot\left(\frac{\partial f(\Delta \eta)}{\partial \Delta \eta}\right)^{T} \frac{W}{2\left|s^{1 / 2}\right|} \zeta^{T} Q \zeta .
\end{gathered}
$$


Finally, apply $s=\dot{\eta}-\dot{\eta}_{r}$ from (9) where $\dot{\eta}_{r}$ is given in (15) yielding

$$
\begin{aligned}
\dot{V}= & -\left(\dot{\eta}-J^{-1}(\eta)(\dot{\eta}-\Delta \eta)-\alpha J^{-1}(\eta) \Delta e_{\eta}\right)\left(\Delta e_{\eta}\right) \\
& +k_{\text {ep }} \max (0, f(\Delta \eta))(\Delta \dot{\eta})^{T}\left(\frac{\partial f(\Delta \eta)}{\partial \Delta \eta}\right)^{T} \\
& -\frac{W}{2\left|s^{1 / 2}\right|} \zeta^{T} Q \zeta .
\end{aligned}
$$

It is obtained that

$$
\dot{V} \leq-\alpha \Delta e_{\eta}{ }^{T} \Delta e_{\eta}-\frac{W}{2\left|s^{1 / 2}\right|} \zeta^{T} Q \zeta \leq 0 .
$$

Since $M_{\eta}$ is uniformly positive definite, $V>0$ and $\dot{V} \leq 0$. Therefore, $V$ is bounded. Then, it also causes $s$ and $\operatorname{EP}(\Delta \eta)$ are bounded. Furthermore, if $W>2 \delta$ and $k>W((5 \delta W+$ $\left.4 \delta^{2}\right) / 2(W-2 \delta)$ ), it causes $Q>0$. In other words, $Q$ is bounded. By applying Barbalat's Lemma, it implies that $\dot{V}$ is negative definite. Therefore, the proposed control (19) for the dynamic system of AUV (3) guarantees the convergence of $\Delta e_{\eta} \rightarrow 0$ and $s \rightarrow 0$ in $t \rightarrow \sim . \Delta e_{\eta} \rightarrow 0$ indicates $f(\Delta \eta) \leq 0$ or it can be said that $\partial f(\Delta \eta) / \partial \Delta \eta$ converges to zero.

\section{Conflict of Interests}

The authors declare that there is no conflict of interests regarding the publication of this paper.

\section{Acknowledgments}

This work was supported in part by the Ministry of Higher Education, Malaysia, and Universiti Teknologi Malaysia under Grants nos. Q.J130000.2509.05H54 and R.J130000.7309.4B156.

\section{References}

[1] Y. Tian and A. Zhang, "Development of a guidance and control system for an underwater plume exploring AUV," in Proceedings of the 8th World Congress on Intelligent Control and Automation (WCICA '10), pp. 6666-6670, Jinan, China, July 2010.

[2] D. R. Yoerger, A. M. Bradley, M. Jakuba, C. R. German, T. Shank, and M. Tivey, "Autonomous and remotely operated vehicle technology for hydrothermal vent discovery, exploration, and sampling," Oceanography, vol. 20, no. 1, pp. 152-161, 2007.

[3] A. Budiyono, "Model predictive control for autonomous underwater vehicle," Indian Journal of Geo-Marine Sciences, vol. 40, no. 2, pp. 191-199, 2011.

[4] L. Lapierre and B. Jouvencel, "Robust nonlinear path-following control of an AUV," IEEE Journal of Oceanic Engineering, vol. 33, no. 2, pp. 89-102, 2008.

[5] S. Bhattacharya, H. Blunck, M. B. Kjargaard, and P. Nurmi, "Robust and energy-efficient trajectory tracking for mobile devices," IEEE Transactions on Mobile Computing, vol. 14, no. 2, pp. 430-443, 2015.

[6] M. Perrier and C. C. de-Wit, "Experimental comparison of PID vs. PID plus nonlinear controller for subsea robots," Autonomous Robots, vol. 3, no. 2-3, pp. 195-212, 1996.
[7] M. Santhakumar and T. Asokan, "A self-tuning proportionalintegral-derivative controller for an autonomous underwater vehicle, Based on Taguchi method," Journal of Computer Science, vol. 6, no. 8, pp. 862-871, 2010.

[8] Q. Li, X. H. Shi, and Z. Q. Kang, "The research of fuzzy-PID control based on grey prediction for AUV," Applied Mechanics and Materials, vol. 246-247, pp. 888-892, 2012.

[9] Q. Chen, T. Chen, and Y. Zhang, "Research of GA-based PID for AUV motion control," in Proceedings of the IEEE International Conference on Mechatronics and Automation (ICMA '09), pp. 4446-4451, August 2009.

[10] E. Roche, O. Sename, D. Simon, and S. Varrier, "A hierarchical varying sampling $H_{\infty}$ control of an AUV," in Proceedings of the 18th IFAC World Congress (IFAC WC '11), pp. 14729-14734, Milano, Italy, August 2011.

[11] Z. H. Ismail and M. W. Dunnigan, "Nonlinear $H_{\infty}$ optimal control scheme for an underwater vehicle with regional function formulation," Journal of Applied Mathematics, vol. 2013, Article ID 732738, 7 pages, 2013.

[12] N. Q. Hoang and E. Kreuzer, "A robust adaptive sliding mode controller for remotely operated vehicles," Technische Mechanik, vol. 28, no. 3-4, pp. 185-193, 2007.

[13] R. Cristi, F. A. Papoulias, and A. J. Healey, "Adaptive sliding mode control of autonomous underwater vehicles in the dive plane," IEEE Journal of Oceanic Engineering, vol. 15, no. 3, pp. $152-160,1990$

[14] H. Akçakaya, H. A. Yildiz, G. Sağlam, and F. Gürleyen, "Sliding mode control of autonomous underwater vehicle," in Proceedings of the 6th International Conference on Electrical and Electronics Engineering (ELECO '09), pp. II332-II336, November 2009.

[15] Z. H. Ismail and M. W. Dunnigan, "A region boundary-based control scheme for an autonomous underwater vehicle," Ocean Engineering, vol. 38, no. 17-18, pp. 2270-2280, 2011.

[16] X. Li and C. C. Cheah, "Adaptive neural network control of robot based on a unified objective bound," IEEE Transactions on Control Systems Technology, vol. 22, no. 3, pp. 1032-1043, 2014.

[17] J. Guo, F.-C. Chiu, and C.-C. Huang, "Design of a sliding mode fuzzy controller for the guidance and control of an autonomous underwater vehicle," Ocean Engineering, vol. 30, no. 16, pp. 21372155, 2003.

[18] G. V. Lakhekar and V. D. Saundarmal, "Novel adaptive fuzzy sliding mode controller for depth control of an underwater vehicles," in Proceedings of the IEEE International Conference on Fuzzy Systems (FUZZ '13), pp. 1-7, Hyderabad, India, July 2013.

[19] J.-H. Li, P.-M. Lee, S. W. Hong, and S. J. Lee, "Stable nonlinear adaptive controller for an autonomous underwater vehicle using neural networks," International Journal of Systems Science, vol. 38, no. 4, pp. 327-337, 2007.

[20] A. Levant, "Higher-order sliding modes, differentiation and output-feedback control," International Journal of Control, vol. 76, no. 9-10, pp. 924-941, 2003.

[21] Y. B. Shtessel, J. A. Moreno, F. Plestan, L. M. Fridman, and A. S. Poznyak, "Super-twisting adaptive sliding mode control: a Lyapunov design," in Proceedings of the 49th IEEE Conference on Decision and Control (CDC '10), pp. 5109-5113, December 2010.

[22] T. Salgado-Jiménez, J.-M. Spiewak, P. Fraisse, and B. Jouvencel, "A robust control algorithm for AUV: based on a high order sliding mode," in Proceedings of the MTTS/IEEE TECHNOOCEAN '04 (OCEANS '04), vol. 1, pp. 276-281, IEEE, Kobe, Japan, November 2004. 
[23] T. I. Fossen and T. I. Fossen, Handbook of Marine Craft Hydrodynamics and Motion Control, John Wiley and Sons, First Edition, New York, 2011.

[24] H. T. Choi, A. Hanai, S. K. Choi, and J. Yuh, "Development of an underwater robot, ODIN-III," in Proceedings of the IEEE/ RSJ International Conference on Intelligent Robots and Systems (IROS '03), vol. 1, pp. 836-841, October 2003.

[25] T. K. Podder and N. Sarkar, "Fault-tolerant control of an autonomous underwater vehicle under thruster redundancy," Robotics and Autonomous Systems, vol. 34, no. 1, pp. 39-52, 2001.

[26] J. A. Moreno and M. Osorio, "A Lyapunov approach to secondorder sliding mode controllers and observers," in Proceedings of the 47th IEEE Conference on Decision and Control (CDC '08), pp. 2856-2861, Cancun, Mexico, December 2008. 


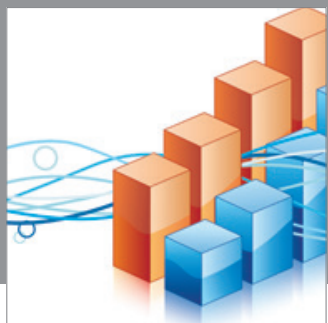

Advances in

Operations Research

mansans

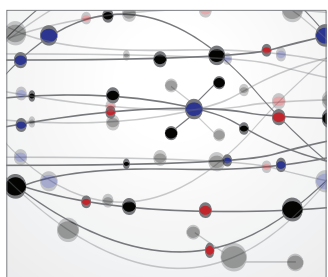

The Scientific World Journal
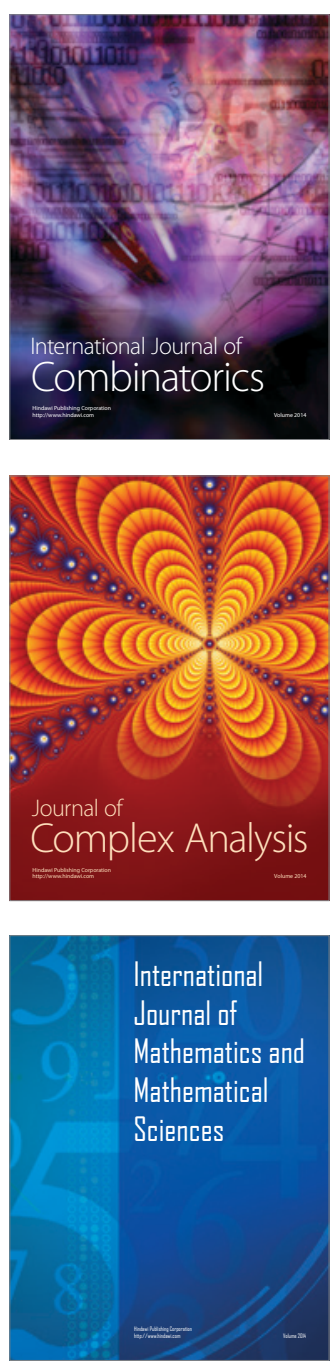
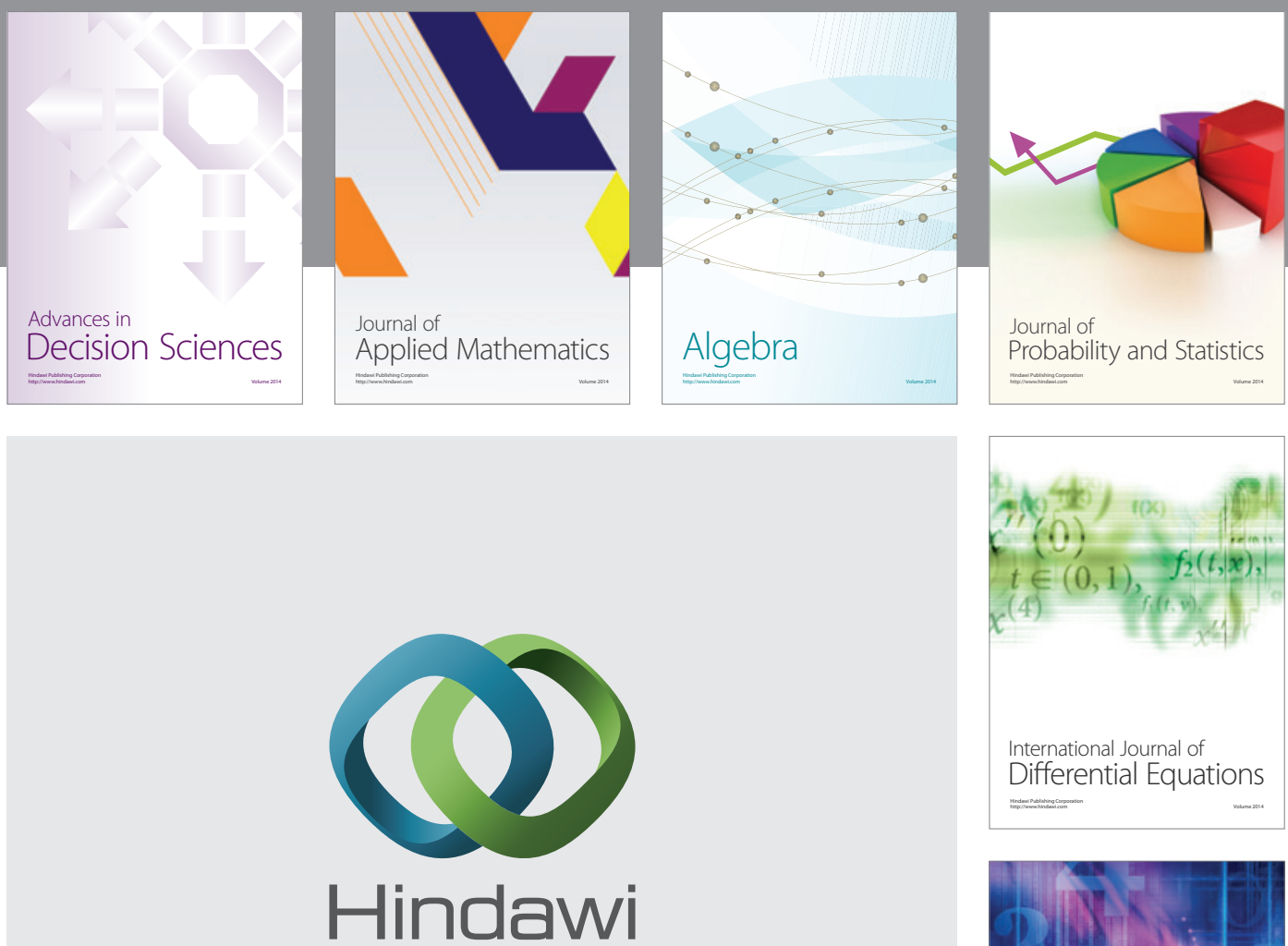

Submit your manuscripts at http://www.hindawi.com
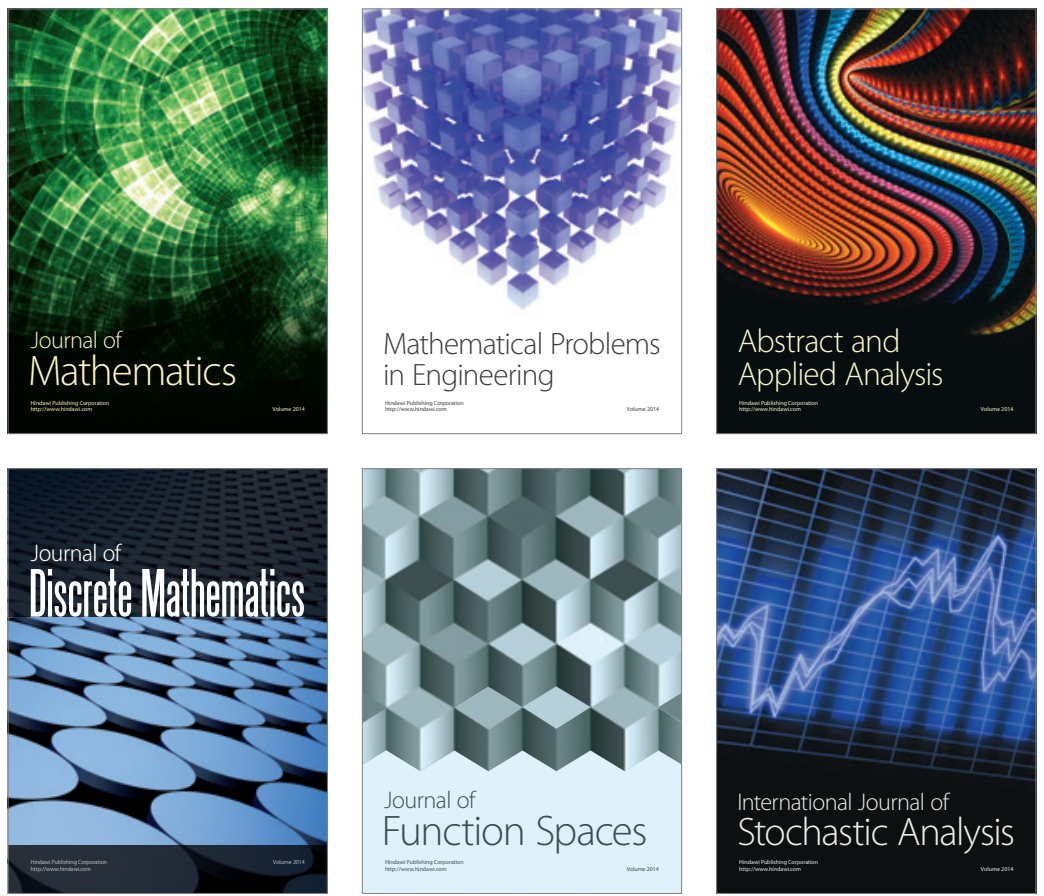

Journal of

Function Spaces

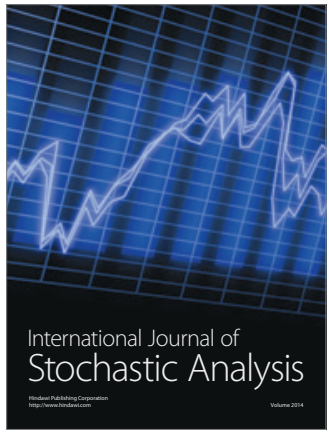

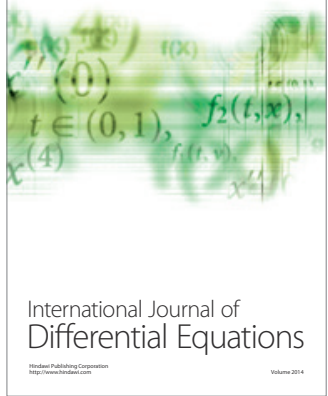
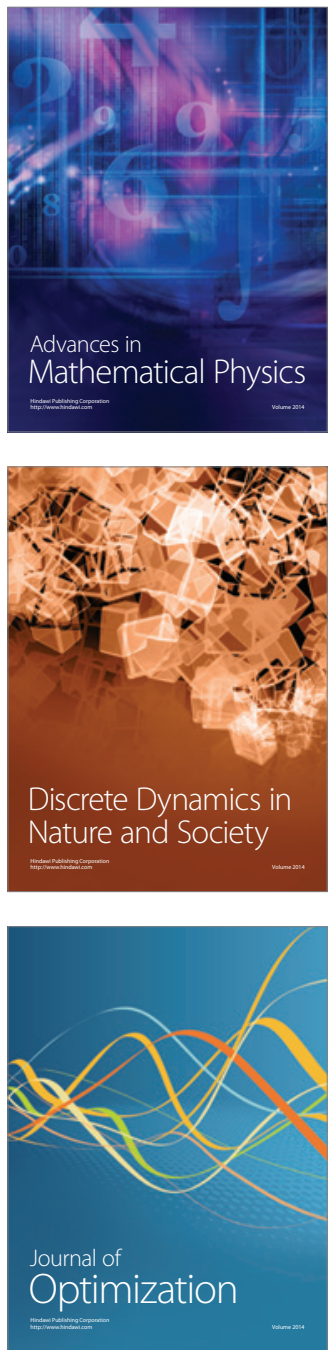